\title{
Succinct Greedy Drawings Do Not Always Exist ${ }^{\star}$
}

\author{
Patrizio Angelini, Giuseppe Di Battista, and Fabrizio Frati \\ Dipartimento di Informatica e Automazione - Roma Tre University, Italy \\ \{angelini,gdb, frati\}adia.uniroma3.it
}

\begin{abstract}
A greedy drawing is a graph drawing containing a distance-decreasing path for every pair of nodes. A path $\left(v_{0}, v_{1}, \ldots, v_{m}\right)$ is distance-decreasing if $d\left(v_{i}, v_{m}\right)<d\left(v_{i-1}, v_{m}\right)$, for $i=1, \ldots, m$. Greedy drawings easily support geographic greedy routing. Hence, a natural and practical problem is the one of constructing greedy drawings in the plane using few bits for representing vertex Cartesian coordinates and using the Euclidean distance as a metric. We show that there exist greedy-drawable graphs that do not admit any greedy drawing in which the Cartesian coordinates have less than a polynomial number of bits.
\end{abstract}

\section{Introduction}

In geographic routing nodes forward packets based on their geographic locations. A very simple geographic routing protocol is greedy routing, in which each node knows its location, the location of its neighbors, and the location of the packet's destination. Based on this information, a node forwards the packet to a neighbor that is closer than itself to the destination's geographic location.

Unfortunately, greedy routing has two weaknesses. First, GPS devices, typically used to determine coordinates, are expensive and increase the energy consumption of the nodes. Second, a bad interaction between the network topology and the node locations can lead to situations in which the communication fails because a void has been reached, i.e., a packet has reached a node whose neighbors are all farther from the destination than the node itself.

A brilliant solution to the greedy routing weaknesses has been proposed by Rao et al., who in [13] proposed a protocol in which nodes are assigned virtual coordinates and the standard greedy routing algorithm is applied relying on such virtual locations rather than on the geographic coordinates. Clearly, virtual coordinates need not to reflect the nodes actual positions and, hence, they can be suitably chosen to guarantee that the greedy routing algorithm succeeds in delivering packets.

After the publication of [13], intense research efforts have been devoted to determine: (i) Which network topologies admit a virtual coordinates assignment such that greedy routing is guaranteed to work. (ii) Which distance metrics, which systems of coordinates, and how many dimensions are suitable for virtual coordinates. (iii) How many bits are needed to represent the vertex coordinates.

From a graph-theoretical point of view, Problem (i) can be stated as follows: Which are the graphs that admit a greedy drawing, i.e., a drawing such that, for every two nodes

\footnotetext{
* This work is partially supported by the Italian Ministry of Research, Grant number RBIP06BZW8, FIRB project “Advanced tracking system in intermodal freight transportation".
} 
$u$ and $v$, there exists a distance-decreasing path from $u$ to $v$ ? A path $\left(v_{0}, v_{1}, \ldots, v_{m}\right)$ is distance-decreasing if $d\left(v_{i}, v_{m}\right)<d\left(v_{i-1}, v_{m}\right)$, for $i=1, \ldots, m$. This formulation of the problem gives a clear perception of how greedy routing can be seen as a "bridge" problem between the theory of routing and Graph Drawing, thus explaining why it attracted attention in both areas.

Concerning drawings in the plane adopting the Euclidean distance, Papadimitriou and Ratajczak [11] showed that $K_{k, 5 k+1}$ has no greedy drawing, for $k \geq 1$. Further, they observed that, if a graph $G$ has a greedy drawing, then any graph containing $G$ as a spanning subgraph has a greedy drawing. Dhandapani [2] showed, with an existential proof based on an application of the Knaster-Kuratowski-Mazurkievicz Theorem [8] to the Schnyder's methodology [14], that every triangulation admits a greedy drawing. Algorithms for constructing greedy drawings of triangulations and triconnected planar graphs have been proposed in [19]. In [9] it is also proved that there exist trees not admitting any greedy drawing.

Concerning Problem (ii), it has been shown that virtual coordinates guarantee greedy routing to work for every tree, and hence for every connected topology, when they can be chosen in the hyperbolic plane [7].

Unfortunately, the above mentioned algorithms construct greedy drawings that are not succinct, i.e., in the worst case they require $\Omega(n \log n)$ bits for representing the vertex coordinates (Problem (iii)). This makes them unsuitable for the motivating application of greedy routing. For solving this drawback, Eppstein and Goodrich [5] proposed an elegant algorithm for greedy routing in the hyperbolic plane representing vertex coordinates with $O(\log n)$ bits. However, the perhaps most natural question of whether greedy drawings can be constructed in the plane using $O(\log n)$ bits for representing vertex Cartesian coordinates and using the Euclidean distance as a metric was, up to now, open. This paper gives a negative answer to the above question.

Theorem 1. For infinitely many $n$, there exists a $(3 n+3)$-node greedy-drawable tree that requires $\Omega\left(b^{n}\right)$ area in any greedy drawing in the plane using the Euclidean distance as a metric, under any finite resolution rule, for some constant $b>1$.

Observe the equivalence between stating the theorem in terms of area requirement of the drawing and in terms of number of bits required for the vertex Cartesian coordinates. Theorem 1 is one of the few results (e.g., [4]) showing that certain families of graph drawings require exponential area. Notice that greedy drawings are a kind of proximity drawings [3], a class of graph drawings, including Euclidean Minimum Spanning Trees [106], for which very little is known about the area requirement [12].

The paper is organized as follows. In Sect. 2 we introduce some definitions and preliminaries; in Sect. 3 we prove that there exists a tree $T_{n}$ requiring exponential area in any greedy drawing; in Sect.4 we show an algorithm for constructing a greedy drawing of $T_{n}$; finally, in Sect.5 we conclude and present some open problems.

\section{Definitions and Preliminaries}

A tree is a connected acyclic graph. The degree of a node is the number of edges incident to it. A leaf is a node with degree 1. A leaf edge is an edge incident to a leaf. A path 
is a tree in which every node other than the leaves has degree 2. A caterpillar is a tree in which the removal of all the leaves and of all the leaf edges yields a path, called spine of the caterpillar, whose nodes and edges are called spine nodes and spine edges, respectively.

A drawing of a graph is a mapping of each node to a distinct point of the plane and of each edge to a Jordan curve between its endpoints. A planar drawing is such that no two edges intersect except, possibly, at common endpoints. A straight-line drawing is such that all the edges are straight-line segments. A planar drawing determines a circular ordering of the edges incident to each node. Two drawings of the same graph are equivalent if they determine the same circular ordering around each node. An embedding is an equivalence class of planar drawings.

The area of a straight-line drawing is the area of its convex hull. The concept of area of a drawing only makes sense for a fixed resolution rule, i.e., a rule that does not allow, e.g., vertices to be arbitrarily close (vertex resolution rule) or edges to be arbitrarily short (edge resolution rule). In fact, without any of such rules, one could construct arbitrarily small drawings with arbitrarily small area. In the following, we derive a lower bound valid under any of such rules. Namely, we prove that, in any greedy drawing of an $n$-node tree $T_{n}$, the ratio between the length of the longest edge and the length of the shortest edge is exponential in $n$, which implies that such a drawing requires exponential area when any resolution rule has been fixed.

We now state some basic properties of the greedy drawings of trees.

The cell of a node $v$ in a drawing is the set of all the points in the plane that are closer to $v$ than to any of its neighbors.

Lemma 1. (Papadimitriou and Ratajczak [11]) A drawing is greedy if and only if the cell of each node $v$ contains no node other than $v$.

We remark that the cell of a leaf node $v$ with parent $u$ is the half-plane containing $v$ and delimited by the axis of segment $\overline{u v}$, where the axis of a segment is the line perpendicular to the segment through its median point.

Lemma 2. Given a greedy drawing $\Gamma$ of a tree $T$, any subtree of $T$ is represented in $\Gamma$ by a greedy drawing.

Proof: Suppose, for a contradiction, that a subtree $T^{\prime}$ of $T$ exists not represented in $\Gamma$ by a greedy drawing. Then, there exist two nodes $u$ and $v$ such that the only path from $u$ to $v$ in $T^{\prime}$ is not distance-decreasing. However, such a path is also the only path from $u$ to $v$ in $T$, a contradiction.

Lemma 3. Given a greedy drawing $\Gamma$ of a tree $T$ and given any edge $(u, v)$ of $T$, the subtree $T^{\prime}$ of $T$ that contains $u$ and that is obtained by removing edge $(u, v)$ from $T$ completely lies in $\Gamma$ in the half-plane containing $u$ and delimited by the axis of segment $\overline{u v}$.

Proof: Suppose, for a contradiction, that there exists a node $w$ of $T^{\prime}$ that lies in $\Gamma$ in the half-plane containing $v$ and delimited by the axis of $\overline{u v}$. Then, $d(v, w)<d(u, w)$. The only path from $v$ to $w$ in $T$ passes through $u$, hence it is not distance-decreasing, a contradiction. 
Lemma 4. Any straight-line greedy drawing of a tree is planar.

Proof: Suppose, for a contradiction, that there exists a tree $T$ admitting a non-planar straight-line greedy drawing $\Gamma$. Let $e_{1}=(u, v)$ and $e_{2}=(w, z)$ be two edges that cross in $\Gamma$. Edges $e_{1}$ and $e_{2}$ are not adjacent, otherwise they would overlap and $\Gamma$ would not be greedy. Then, there exists an edge $e_{3} \neq e_{1}, e_{2}$ in the only path connecting $u$ to $w$. Lemma 3 implies that $e_{1}$ and $e_{2}$ lie in distinct half-planes delimited by the axis of the segment representing $e_{3}$, hence they do not cross, a contradiction.

Corollary 1. Consider a greedy drawing $\Gamma$ of a tree $T$. For each edge, remove its drawing from $\Gamma$ and substitute it with a straight-line segment connecting its endpoints. The resulting drawing is a straight-line planar greedy drawing of $T$.

Because of Lemma 4 and of Corollary 1 in order to prove Theorem 1 we can restrict the attention to planar straight-line greedy drawings. In the following, all considered drawings will be planar and straight-line.

Lemma 5. In any greedy drawing of a tree $T$, the angle between two adjacent segments is strictly greater than $60^{\circ}$.

Proof: Consider any greedy drawing of $T$ in which the angle between two adjacent segments $\overline{w_{1} w_{2}}$ and $\overline{w_{2} w_{3}}$ is no more than $60^{\circ}$. Then, $\left|\overline{w_{1} w_{3}}\right| \leq\left|\overline{w_{1} w_{2}}\right|$ or $\left|\overline{w_{1} w_{3}}\right| \leq$ $\left|\overline{w_{2} w_{3}}\right|$, say $\left|\overline{w_{1} w_{3}}\right| \leq\left|\overline{w_{2} w_{3}}\right|$. Since $d\left(w_{1}, w_{3}\right) \leq d\left(w_{2}, w_{3}\right)$, the unique path $\left(w_{1}, w_{2}, w_{3}\right)$ from $w_{1}$ to $w_{3}$ in $T$ is not distance-decreasing.

In the following we define a family of trees with $3 n+3$ nodes, for every $n \geq 2$, that will be exploited in order to prove Theorem 1 Refer to Fig. 1.

Definition 1. Let $T_{n}$ be a caterpillar with spine $\left(v_{1}, v_{2}, \ldots, v_{n}\right)$ such that $v_{1}$ has degree 5 and $v_{i}$ has degree 4 , for each $i=2,3, \ldots, n$. Let $a_{1}, b_{1}, c_{1}$, and $d_{1}$ be the leaves of $T_{n}$ adjacent to $v_{1}$, let $a_{i}$ and $b_{i}$ be the leaves of $T_{n}$ adjacent to $v_{i}$, for $i=2,3, \ldots, n-1$, and let $a_{n}, b_{n}$, and $c_{n}$ be the leaves of $T_{n}$ adjacent to $v_{n}$.

Distinct embeddings of $T_{n}$ differ for the order of the edges incident to the spine nodes. More precisely, the clockwise order of the edges incident to each node $v_{i}$ is one of the following: 1) $\left(v_{i-1}, v_{i}\right)$, then a leaf edge, then $\left(v_{i}, v_{i+1}\right)$, then a leaf edge: $v_{i}$ is a central node (node $v_{n}$ in Fig. 1 b); 2) $\left(v_{i-1}, v_{i}\right)$, then two leaf edges, then $\left(v_{i}, v_{i+1}\right)$ : $v_{i}$ is a bottom node (node $v_{2}$ in Fig. 1 b); or 3$)\left(v_{i-1}, v_{i}\right)$, then $\left(v_{i}, v_{i+1}\right)$, then two leaf edges: $v_{i}$ is a top node (node $v_{3}$ in Fig. 1, b). Node $v_{1}$ is considered as a central node.

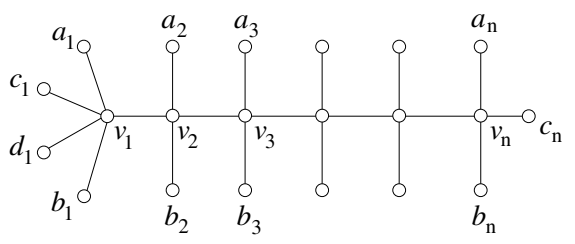

(a)

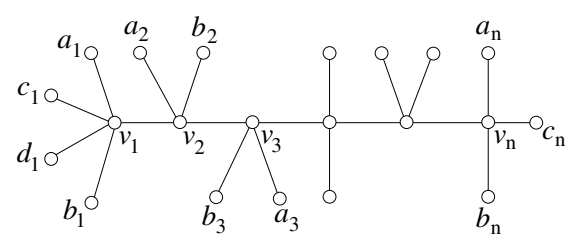

(b)

Fig. 1. Two embeddings of caterpillar $T_{n}$. In (a) all the spine nodes are central nodes. In (b) node $v_{2}$ is a bottom node and node $v_{3}$ is a top node. 


\section{The Lower Bound}

In this section we prove that any greedy drawing of $T_{n}$ requires exponential area.

The proof is based on the following intuitions: (i) For any central node $v_{i}$ there exists a "small" convex region containing all the spine nodes $v_{j}$, with $j>i$, and their adjacent leaves (Lemma6). (ii) Almost all the spine nodes are central nodes (Lemma 8). (iii) The slopes of edges $\left(v_{i}, a_{i}\right),\left(v_{i}, v_{i+1}\right)$, and $\left(v_{i}, b_{i}\right)$ incident to a central node $v_{i}$ are in a certain range, which is more restricted for the edges incident to $v_{i+1}$ than for those incident to $v_{i}$ (Lemma 6). (iv) If the angle between $\left(v_{i}, a_{i}\right)$ and $\left(v_{i}, b_{i}\right)$ is too small, then $v_{j}, a_{j}$, and $b_{j}$, with $j \geq i+2$, can not be drawn (Lemma 10). (v) If both the angles between $\left(v_{i}, a_{i}\right)$ and $\left(v_{i}, b_{i}\right)$, and between $\left(v_{i+1}, a_{i+1}\right)$ and $\left(v_{i+1}, b_{i+1}\right)$ are large enough, then the ratio between the length of the edges incident to $v_{i}$ and the length of the edges incident to $v_{i+1}$ is constant (Lemma 9).

First, we discuss some properties of the slopes of the edges in the drawing. Second, we argue about the exponential decrease of the edge lengths.

\subsection{Slopes}

Consider any drawing of $v_{1}$ and of its adjacent leaves; rename such leaves so that the counter-clockwise order of the vertices around $v_{1}$ is $a_{1}, c_{1}, d_{1}, b_{1}$, and $v_{2}$.

In the following, when we refer to an angle $\widehat{v_{1} v_{2} v_{3}}$, we mean the angle that brings the half-line from $v_{2}$ through $v_{1}$ to coincide with the half-line from $v_{2}$ through $v_{3}$ by a counter-clockwise rotation.

Property 1. $\widehat{b_{1} v_{1} a_{1}}<180^{\circ}$.

Proof: By Lemma 5, $\widehat{a_{1} v_{1} c_{1}}>60^{\circ}, \widehat{c_{1} v_{1} d_{1}}>60^{\circ}$, and $\widehat{d_{1} v_{1} b_{1}}>60^{\circ}$.

Now we argue that, for any central node $v_{i}$, there exists a "small" convex region that contains all the spine nodes $v_{j}$, with $j>i$, and their adjacent leaves.

Let $v_{i}$ be a central node and suppose that $\widehat{b_{i} v_{i} a_{i}}<180^{\circ}$. Denote by $R_{i}$ the convex region delimited by $\overline{v_{i} a_{i}}$, by $\overline{v_{i} b_{i}}$, and by the axes of such segments (see Fig. 2. b). Denote by $p_{i}$ the intersection between the axes of $\overline{v_{i} a_{i}}$ and $\overline{v_{i} b_{i}}$, and by $h_{i}^{a}\left(h_{i}^{b}\right)$ the midpoint of $\overline{v_{i} a_{i}}$ (resp. $\overline{v_{i} b_{i}}$ ).

Assume that $x\left(a_{i}\right)=x\left(b_{i}\right), x\left(v_{i}\right)<x\left(a_{i}\right)$, and $y\left(a_{i}\right)>y\left(b_{i}\right)$. Such a setting can be achieved without loss of generality up to a rotation/mirroring of the drawing and a renaming of the leaves. In the following, whenever a central node $v_{i}$ is considered, the drawing is rotated/mirrored and the leaves adjacent to $v_{i}$ are renamed so that $x\left(a_{i}\right)=$ $x\left(b_{i}\right), x\left(v_{i}\right)<x\left(a_{i}\right)$, and $y\left(a_{i}\right)>y\left(b_{i}\right)$.

Let $\operatorname{slope}(u, v)$ be the angle bringing the half-line from $u$ directed downward to coincide with the half-line from $u$ through $v$ by a counter-clockwise rotation (see Fig. 2]a). Further, let slope $_{\perp}(u, v)$ be equal to $\operatorname{slope}(u, v)-90^{\circ}$. We observe the following:

Property 2. $\operatorname{slope}\left(v_{i}, b_{i}\right)<\operatorname{slope}_{\perp}\left(b_{i}, p_{i}\right)<\operatorname{slope}_{\perp}\left(p_{i}, a_{i}\right)<\operatorname{slope}\left(v_{i}, a_{i}\right)$.

Proof: Inequality slope $\left(v_{i}, b_{i}\right)<\operatorname{slope}_{\perp}\left(b_{i}, p_{i}\right)$ (and analogously $\operatorname{slope}_{\perp}\left(p_{i}, a_{i}\right)<$ $\left.\operatorname{slope}\left(v_{i}, a_{i}\right)\right)$ holds since $\operatorname{slope}\left(h_{i}^{b}, p_{i}\right)<\operatorname{slope}\left(b_{i}, p_{i}\right)$. Inequality $\operatorname{slope}_{\perp}\left(b_{i}, p_{i}\right)<$ slope $_{\perp}\left(p_{i}, a_{i}\right)$ holds by assumption. 


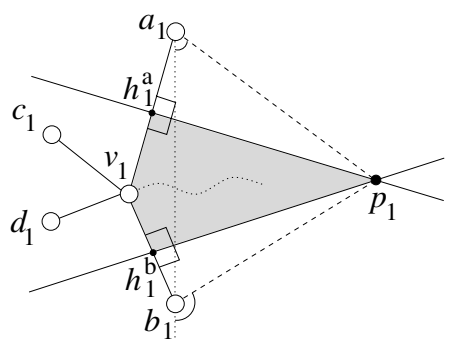

(a)

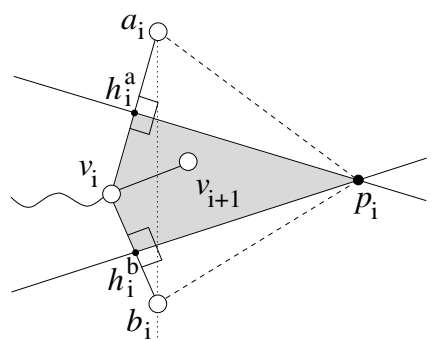

(b)

Fig. 2. (a) Region $R_{1}$ contains the drawing of $T_{n} \backslash\left\{a_{1}, b_{1}, c_{1}, d_{1}, v_{1}\right\}$. The slopes of $\overline{a_{1} p_{1}}$ and $\overline{b_{1} p_{1}}$ are shown. (b) Region $R_{i}$ contains the drawing of path $\left(v_{i+1}, v_{i+2}, \ldots, v_{n}\right)$ and of its adjacent leaves.

Lemma 6. Suppose that $v_{i}$ is a central node. Then, the following properties hold: (i) $\widehat{b_{i} v_{i} a_{i}}<180^{\circ}$; (ii) the drawing of path $\left(v_{i+1}, v_{i+2}, \ldots, v_{n}\right)$ and of its adjacent leaves lies in $R_{i}$; and (iii) any edge $\left(v_{j}, x\right)$, where $x \in\left\{a_{j}, b_{j}, v_{j+1}\right\}$ with $j>i$, is such that slope $\perp_{\perp}\left(b_{i}, p_{i}\right)<\operatorname{slope}\left(v_{j}, x\right)<$ slope $_{\perp}\left(p_{i}, a_{i}\right)$. See Fig. 3. $a$.

Proof: When $i=1$, Property 1 ensures property (i). Further, Lemma 1 ensures property (ii), that is, the drawing of $T_{n} \backslash\left\{a_{1}, b_{1}, c_{1}, d_{1}, v_{1}\right\}$ lies in $R_{1}$ (see Fig. 2] a). In order to prove property (iii), suppose, for a contradiction, that an edge $\left(v_{j}, x\right)$ exists, where $x \in$ $\left\{a_{j}, b_{j}, v_{j+1}\right\}$ with $j>1$, such that $\operatorname{slope}_{\perp}\left(b_{1}, p_{1}\right)<\operatorname{slope}\left(v_{j}, x\right)<\operatorname{slope}_{\perp}\left(p_{1}, a_{1}\right)$ does not hold. Then, it is easy to see that the half-plane delimited by the axis of $\overline{v_{j} x}$ and containing $x$ also contains at least one out of $a_{1}, v_{1}$, and $b_{1}$, thus providing a contradiction to the greediness of the drawing, by Lemma 3

By induction, suppose that properties (i), (ii), and (iii) of the lemma hold for some $i$. Let $k$ be the smallest index greater than $i$ such that $v_{k}$ is a central node. Then, by property (iii) of the inductive hypothesis and by Property 2 slope $\left(v_{i}, b_{i}\right)<\operatorname{slope}_{\perp}\left(b_{i}, p_{i}\right)$ $<\operatorname{slope}\left(v_{k}, b_{k}\right)<\operatorname{slope}\left(v_{k}, a_{k}\right)<\operatorname{slope}_{\perp}\left(p_{i}, a_{i}\right)<\operatorname{slope}\left(v_{i}, a_{i}\right)$ holds, which implies $\widehat{b_{k} v_{k} a_{k}}<\widehat{b_{i} v_{i} a_{i}}<180^{\circ}$, and property (i) of the lemma follows for $k$.

By Lemma 4 the drawing is planar; by Lemma 1, the cells of $a_{k}$ and $b_{k}$ do not contain any node other than $a_{k}$ and $b_{k}$, respectively. Hence, if a node $u$ is in $R_{k}$, then no node of any subtree of $T_{n}$ containing $u$ and not containing $v_{k}$ lies outside $R_{k}$. Thus, $v_{k-1}$ does not lie in $R_{k}$ (since a subtree of $T_{n}$ exists containing $v_{k-1}, v_{i}$, and not containing $v_{k}$ ); since $v_{k}$ is a central node, then $v_{k+1}$ lies on the opposite side of $v_{k-1}$ with respect to the path composed of edges $\left(v_{k}, a_{k}\right)$ and $\left(v_{k}, b_{k}\right)$. Hence, $v_{k+1}$ (and path $\left(v_{k+1}, v_{k+2}, \ldots, v_{n}\right)$ together with its adjacent leaves) lies inside $R_{k}$, and property (ii) of the lemma follows for $k$.

Property (iii) can be proved analogously as in the base case, by implicitly exploiting that properties (i) and (ii) hold for $k$. Namely, if $\operatorname{slope}_{\perp}\left(b_{k}, p_{k}\right)<\operatorname{slope}\left(v_{j}, x\right)<$ slope $\perp_{\perp}\left(p_{k}, a_{k}\right)$ does not hold, for some edge $\left(v_{j}, x\right)$ with $j>k$, then the half-plane delimited by the axis of $\overline{v_{j} x}$ and containing $x$ also contains at least one out of $a_{k}, v_{k}$, and $b_{k}$, thus implying that the drawing is not greedy, by Lemma 3 


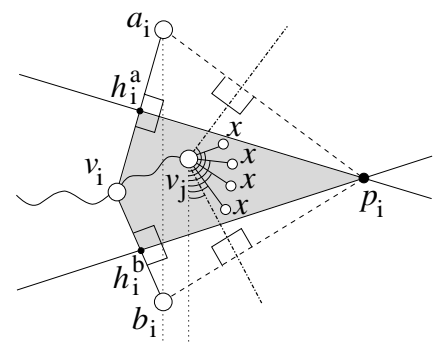

(a)

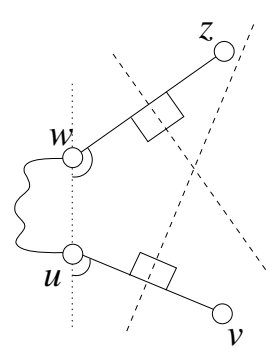

(b)

Fig. 3. (a) Possible slopes for an edge $\left(v_{j}, x\right)$. (b) Illustration for the proof of Lemma7

Now we give a general property of a greedy drawing of a tree. Consider two edges $(u, v)$ and $(w, z)$ such that the path from $u$ to $w$ does not contain $v$ and $z$. Suppose that $v$ and $z$ lie in the same half-plane delimited by the line through $u$ and $w$. Suppose, without loss of generality up to a rotation/mirroring of the drawing, that $x(u)=x(w)$, $y(u)<y(w)$, and $0^{\circ}<\operatorname{slope}(u, v)$, slope $(w, z)<180^{\circ}$. See Fig. 3. b.

Lemma 7. $\operatorname{slope}(u, v)<\operatorname{slope}(w, z)$.

Proof: Suppose, for a contradiction, that $\operatorname{slope}(u, v) \geq \operatorname{slope}(w, z)$. Then, either $v$ lies in the half-plane delimited by the axis of $\overline{w z}$ and containing $z$, or $z$ lies in the half-plane delimited by the axis of $\overline{u v}$ and containing $v$. Hence, by Lemma 2 the drawing is not greedy.

\subsection{Exponential Decreasing Edge Lengths}

Now we are ready to go in the mainstream of the proof that any greedy drawing of $T_{n}$ requires exponential area. Such a proof is in fact based on the following three lemmata. The first one states that a linear number of spine nodes are central nodes, in any greedy drawing of $T_{n}$.

Lemma 8. Suppose that $v_{i}$ is a central node, for some $i \leq n-3$. Then, $v_{i+1}$ is a central node.

Proof: Refer to Fig. 4. Suppose, for a contradiction, that $v_{i+1}$ is not a central node. Suppose that $v_{i+1}$ is a top node, the case in which it is a bottom node being analogous. Rename the leaves adjacent to $v_{i+1}$ in such a way that the counter-clockwise order of the neighbors of $v_{i+1}$ is $v_{i}, b_{i+1}, a_{i+1}$, and $v_{i+2}$. By property (i) of Lemma 6 . $\widehat{b_{i} v_{i} a_{i}}<180^{\circ}$. By property (iii) of Lemma 6 by Property 2 , and by the assumption that $v_{i+1}$ is a top node, slope $\left(v_{i}, b_{i}\right)<\operatorname{slope}\left(v_{i+1}, b_{i+1}\right)<\operatorname{slope}\left(v_{i+1}, a_{i+1}\right)<$ $\operatorname{slope}\left(v_{i+1}, v_{i+2}\right)<\operatorname{slope}\left(v_{i}, a_{i}\right)$. By Lemma $5, b_{i+1} \widehat{v_{i+1}} a_{i+1}>60^{\circ}$. It follows that $a_{i+1}{\widehat{v_{i+1}}}_{i+2}<120^{\circ}$.

Suppose that $v_{i+2}$ is a central node (a top node; a bottom node). Rename the leaves adjacent to $v_{i+2}$ in such a way that the counter-clockwise order of the neighbors of $v_{i+2}$ is $v_{i+1}, b_{i+2}, v_{i+3}$, and $a_{i+2}$ (resp. $v_{i+1}, b_{i+2}, a_{i+2}$, and $v_{i+3} ; v_{i+1}, v_{i+3}, b_{i+2}$, and 


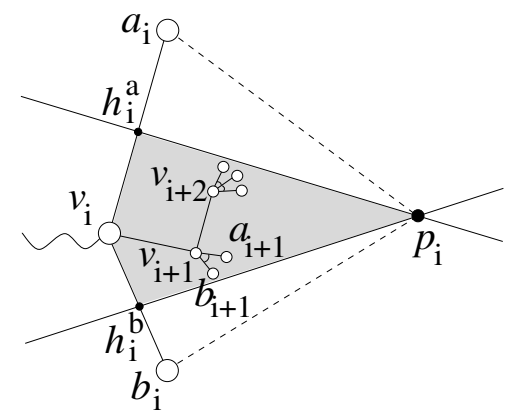

Fig. 4. Illustration for the proof of Lemma 8

$\left.a_{i+2}\right)$. Notice that node $v_{i+3}$ exists since $i \leq n-3$. By Lemma 7 slope $\left(v_{i+2}, b_{i+2}\right)>$ $\operatorname{slope}\left(v_{i+1}, a_{i+1}\right)$ (resp. slope $\left(v_{i+2}, b_{i+2}\right)>\operatorname{slope}\left(v_{i+1}, a_{i+1}\right) ; \operatorname{slope}\left(v_{i+2}, v_{i+3}\right)>$ $\left.\operatorname{slope}\left(v_{i+1}, a_{i+1}\right)\right)$. By property (iii) of Lemma 6 slope $\left(v_{i+2}, a_{i+2}\right)<\operatorname{slope}\left(v_{i}, a_{i}\right)$ (resp. slope $\left.\left(v_{i+2}, v_{i+3}\right)<\operatorname{slope}\left(v_{i}, a_{i}\right) ; \operatorname{slope}\left(v_{i+2}, a_{i+2}\right)<\operatorname{slope}\left(v_{i}, a_{i}\right)\right)$. It follows that $b_{i+2} \widehat{v_{i+2} a_{i+2}}<120^{\circ}$ (resp. $b_{i+2}{\widehat{v_{i+2}}}_{i+3}<120^{\circ} ; v_{i+3} \widehat{v_{i+2}} a_{i+2}<120^{\circ}$ ), hence at least one of $b_{i+2} \widehat{v_{i+2} v_{i+3}}$ and $v_{i+3} \widehat{v_{i+2} a_{i+2}}$ (resp. of $b_{i+2} \widehat{v_{i+2}} a_{i+2}$ and $a_{i+2}{\widehat{v_{i+2}}}_{i+3}$; of $v_{i+3}{\widehat{v_{i+2}}}_{i+2}$ and $b_{i+2}{\widehat{v_{i+2}}}_{i+2}$ ) is less than $60^{\circ}$. By Lemma 5 the drawing is not greedy.

The next lemma shows that, if the angles $\widehat{b_{i} v_{i} a_{i}}$ incident to each central node $v_{i}$ are large enough, then the sum of the lengths of $\overline{v_{i} a_{i}}$ and $\overline{v_{i} b_{i}}$ decreases exponentially in the number of considered central nodes.

Lemma 9. Let $v_{i}$ be a central node, with $i \leq n-3$. Suppose that both the angles $\widehat{b_{i} v_{i} a_{i}}$ and $b_{i+1} \widehat{v_{i+1} a_{i+1}}$ are greater than $150^{\circ}$. Then, the following inequality holds: $\left|\overline{v_{i+1} a_{i+1}}\right|+\left|\overline{v_{i+1} b_{i+1}}\right| \leq\left(\left|\overline{v_{i} a_{i}}\right|+\left|\overline{v_{i} b_{i}}\right|\right) / \sqrt{3}$.

Proof: Refer to Fig. 5]a. By Lemma 8, $v_{i+1}$ is a central node. Denote by $l\left(v_{i+1}\right)$ the vertical line through $v_{i+1}$ and denote by $l\left(h_{i}^{a}\right)$ and $l\left(h_{i}^{b}\right)$ the horizontal lines through $h_{i}^{a}$ and $h_{i}^{b}$, respectively.

By property (iii) of Lemma 6, we have that $\operatorname{slope}_{\perp}\left(b_{i}, p_{i}\right)<\operatorname{slope}\left(v_{i+1}, b_{i+1}\right)<$ $\operatorname{slope}\left(v_{i+1}, a_{i+1}\right)<\operatorname{slope}_{\perp}\left(p_{i}, a_{i}\right)$. Hence, by Property 2 , we have $\operatorname{slope}\left(v_{i}, b_{i}\right)<$ $\operatorname{slope}\left(v_{i+1}, b_{i+1}\right)<\operatorname{slope}\left(v_{i+1}, a_{i+1}\right)<\operatorname{slope}\left(v_{i}, a_{i}\right)$. It follows that both $a_{i+1}$ and $b_{i+1}$ lie in the half-plane delimited by $l\left(v_{i+1}\right)$ and not containing $v_{i}$. Denote by $d_{i+1}^{a}$ $\left(d_{i+1}^{b}\right)$ the intersection point between $l\left(v_{i+1}\right)$ and $l\left(h_{i}^{a}\right)$ (resp. and $l\left(h_{i}^{b}\right)$ ). Observe that $\left|\overline{d_{i+1}^{b} d_{i+1}^{a}}\right|<\left(\left|\overline{v_{i} b_{i}}\right|+\left|\overline{v_{i} a_{i}}\right|\right) / 2$. Denote by $f_{i+1}^{a}$ (by $\left.f_{i+1}^{b}\right)$ the intersection point between $l\left(h_{i}^{a}\right)$ and the line through $v_{i+1}$ and $a_{i+1}$ (resp. between $l\left(h_{i}^{b}\right)$ and the line through $v_{i+1}$ and $\left.b_{i+1}\right)$. Clearly, $\left|\overline{v_{i+1} a_{i+1}}\right|<\left|\overline{v_{i+1} f_{i+1}^{a}}\right|$ and $\left|\overline{v_{i+1} b_{i+1}}\right|<\left|\overline{v_{i+1} f_{i+1}^{b}}\right|$. Angles $d_{i+1}^{b} \widehat{v_{i+1}} f_{i+1}^{b}$ and $f_{i+1}^{a} \widehat{v_{i+1}} d_{i+1}^{a}$ are each less than $30^{\circ}$, namely such angles sum up to an angle which is $180^{\circ}$ minus $f_{i+1}^{b} \widehat{v_{i+1}} f_{i+1}^{a}$, which by hypothesis is greater than $150^{\circ}$. Hence, $\left|\overline{v_{i+1} a_{i+1}}\right|<\left|\overline{v_{i+1} f_{i+1}^{a}}\right|<\left|\overline{v_{i+1} d_{i+1}^{a}}\right| / \cos (30)$ and $\left|\overline{v_{i+1} b_{i+1}}\right|<$ $\left|\overline{v_{i+1} f_{i+1}^{b}}\right|<\overline{v_{i+1} d_{i+1}^{b}} \mid / \cos (30)$. 


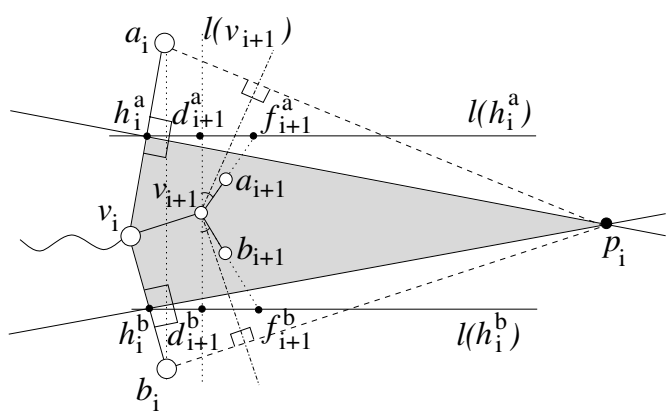

(a)

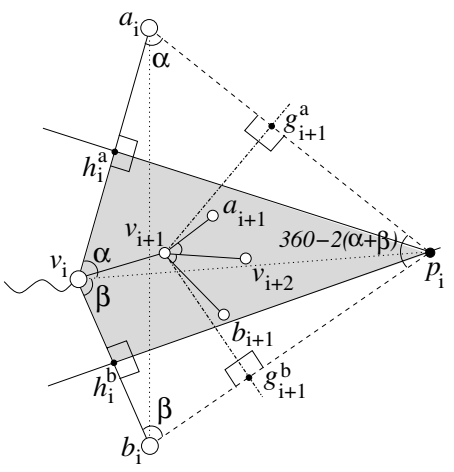

(b)

Fig. 5. Illustrations for the proofs of Lemma9 (a) and Lemma 10 (b)

It follows that $\left|\overline{v_{i+1} a_{i+1}}\right|+\left|\overline{v_{i+1} b_{i+1}}\right|<\left(\left|\overline{v_{i+1} d_{i+1}^{a}}\right|+\left|\overline{v_{i+1} d_{i+1}^{b}}\right|\right) / \cos (30)<$ $2\left(\left|\overline{v_{i} b_{i}}\right|+\left|\overline{v_{i} a_{i}}\right|\right) / 2 \sqrt{3}$, thus proving the lemma.

The next lemma shows that having large angles incident to central nodes is unavoidable for almost all central nodes.

Lemma 10. No central node $v_{i}$, with $i \leq n-3$, is incident to an angle $\widehat{b_{i} v_{i} a_{i}}$ that is less than or equal to $150^{\circ}$.

Proof: Refer to Fig. 5]b. Suppose, for a contradiction, that there exists a central node $v_{i}$, with $i \leq n-3$, that is incident to an angle $\widehat{b_{i} v_{i} a_{i}} \leq 150^{\circ}$. Denote by $\alpha$ and $\beta$ the angles $\widehat{p_{i} v_{i} a_{i}}$ and $\widehat{b_{i} v_{i} p_{i}}$, respectively. Since triangles $\left(v_{i}, p_{i}, h_{i}^{a}\right)$ and $\left(a_{i}, p_{i}, h_{i}^{a}\right)$ are congruent, $\widehat{v_{i} a_{i} p_{i}}=\alpha$. Analogously, $\widehat{v_{i} b_{i} p_{i}}=\beta$. Summing up the angles of quadrilateral $\left(v_{i}, a_{i}, p_{i}, b_{i}\right)$, we get $\widehat{a_{i} p_{i} b_{i}}=360^{\circ}-2(\alpha+\beta)$.

By Lemma 8, $v_{i+1}$ is a central node. Consider the line through $v_{i+1}$ orthogonal to $\overline{a_{i} p_{i}}$ and denote by $g_{i+1}^{a}$ the intersection point between such a line and $\overline{a_{i} p_{i}}$. Further, consider the line through $v_{i+1}$ orthogonal to $\overline{b_{i} p_{i}}$ and denote by $g_{i+1}^{b}$ the intersection point between such a line and $\overline{b_{i} p_{i}}$. By property (iii) of Lemma $6 \operatorname{slope}_{\perp}\left(b_{i}, p_{i}\right)<$ $\operatorname{slope}\left(v_{i+1}, b_{i+1}\right)<\operatorname{slope}\left(v_{i+1}, a_{i+1}\right)<\operatorname{slope}_{\perp}\left(p_{i}, a_{i}\right)$. Hence, $b_{i+1}{\widehat{v_{i+1}}}_{i+1}<$ $g_{i+1}^{b} \widehat{v_{i+1}} g_{i+1}^{a}$. Further, $g_{i+1}^{b} \widehat{v_{i+1}} g_{i+1}^{a}=2 \alpha+2 \beta-180^{\circ}$, as can be derived by considering quadrilateral $\left(g_{i+1}^{b}, v_{i+1}, g_{i+1}^{a}, p_{i}\right)$. Since, by hypothesis, $\alpha+\beta \leq 150^{\circ}$, we have $b_{i+1}{\widehat{v_{i+1}}}_{i+1}<g_{i+1}^{b} \widehat{v_{i+1}} g_{i+1}^{a}=2 \alpha+2 \beta-180^{\circ} \leq 120^{\circ}$. However, since $v_{i+1}$ is a central node, edge $\left(v_{i+1} v_{i+2}\right)$, that exists since $i \leq n-3$, cuts angle $b_{i+1} \widehat{v_{i+1}} a_{i+1}$. It follows that at least one of angles $b_{i+1} \widehat{v_{i+1} v_{i+2}}$ and $v_{i+2} \widehat{v_{i+1}} a_{i+1}$ is less than $60^{\circ}$. By Lemma 5 the drawing is not greedy.

The previous lemmata immediately imply an exponential lower bound between the ratio of the lengths of the longest and the shortest edge of the drawing. Namely, node $v_{1}$ is a central node. By Lemma $8, v_{i}$ is a central node, for $i=2, \ldots, n-3$. By Lemma 10, angle $\widehat{b_{i} v_{i} a_{i}}>150^{\circ}$, for each $i \leq n-3$. Hence, by Lemma $9\left|\overline{v_{i+1} a_{i+1}}\right|+\left|\overline{v_{i+1} b_{i+1}}\right| \leq$ 


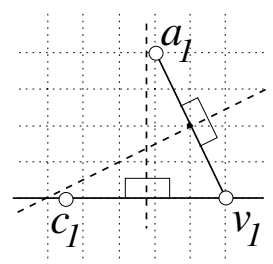

(a)

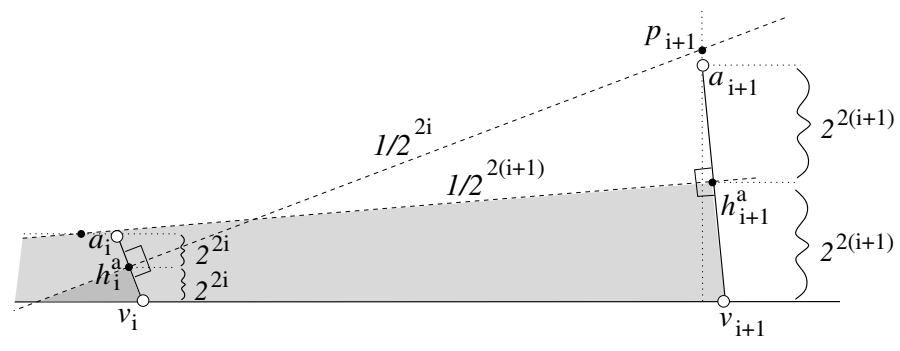

(b)

Fig. 6. Illustrations for the algorithm to construct a greedy drawing of $T_{n}$. (a) Base case. (b) Inductive case.

$\left(\left|\overline{v_{i} a_{i}}\right|+\left|\overline{v_{i} b_{i}}\right|\right) / \sqrt{3}$, for each $i \leq n-4$; it follows that $\left|\overline{v_{n-3} a_{n-3}}\right|+\left|\overline{v_{n-3} b_{n-3}}\right| \leq$ $\left(\left|\overline{v_{1} a_{1}}\right|+\left|\overline{v_{1} b_{1}}\right|\right) /(\sqrt{3})^{n-4}$. Since one out of $\overline{v_{1} a_{1}}$ and $\overline{v_{1} b_{1}}$, say $\overline{v_{1} a_{1}}$, has length at least half of $\left|\overline{v_{1} a_{1}}\right|+\left|\overline{v_{1} b_{1}}\right|$, and since one out of $\overline{v_{n-3} a_{n-3}}$ and $\overline{v_{n-3} b_{n-3}}$, say $\overline{v_{n-3} a_{n-3}}$, has length at most half of $\left|\overline{v_{n-3} a_{n-3}}\right|+\left|\overline{v_{n-3} b_{n-3}}\right|$, then $\left|\overline{v_{1} a_{1}}\right| /\left|\overline{v_{n-3} a_{n-3}}\right| \geq$ $\frac{1}{9}(\sqrt{3})^{n}$, thus implying the claimed lower bound.

\section{Drawability of $T_{n}$}

In Sect. 3 we have shown that any greedy drawing of $T_{n}$ requires exponential area. Since in [119] it has been shown that there exist trees that do not admit any greedy drawing, one might ask whether the lower bound refers to a greedy-drawable tree or not. Of course, if $T_{n}$ were not drawable, then the lower bound would not make sense. In this section we show that $T_{n}$ admits a greedy drawing by providing a drawing algorithm, using a supporting exponential-size grid.

Since the algorithm draws the spine nodes in the order they appear on the spine with the degree- 5 node as the last node, we revert the indices of the nodes with respect to Sects. 2 and 3, that is, node $v_{i}$ of $T_{n}$ is now node $v_{n-i+1}$.

The algorithm constructs a drawing of $T_{n}$ in which all the spine nodes $v_{i}$ are central nodes lying on the horizontal line $y=0$. Since each leaf node $a_{i}$ is drawn above line $y=0$ and $b_{i}$ is placed on the symmetrical point of $a_{i}$ with respect to such a line, we only describe, for each $i=1, \ldots, n$, how to draw $v_{i}$ and $a_{i}$.

In order to deal with drawings that lie on a grid, in this section we denote by $\Delta_{y} / \Delta_{x}$ the slope of a line (of a segment), meaning that whenever there is a horizontal distance $\Delta_{x}$ between two nodes of such a line (of such a segment), then their vertical distance is $\Delta_{y}$.

The drawing is constructed by means of an inductive algorithm. In the base case, place $v_{1}$ at $(0,0), h_{1}^{a}$ at $(-1,2), a_{1}$ at $(-2,4)$, and $c_{1}$ at $(-9 / 2,0)$ (see Fig. 6. a). At step $i$ of the algorithm suppose, by inductive hypothesis, that: (i) The drawing of path $\left(v_{1}, v_{2}, \ldots, v_{i}\right)$ with its leaf nodes $a_{1}, a_{2}, \ldots, a_{i}$ is greedy, and (ii) $y\left(v_{i}\right)=0, y\left(h_{i}^{a}\right)=$ $2^{2 i}, y\left(a_{i}\right)=2^{2 i+1}$, and $x\left(v_{i}\right)-x\left(h_{i}^{a}\right)=x\left(h_{i}^{a}\right)-x\left(a_{i}\right)=1$.

From the above inductive hypothesis it follows that the slope of segment $\overline{v_{i} a_{i}}$ is $-2^{2 i} / 1$ and the slope of its axis is $1 / 2^{2 i}$. We show step $i+1$ of the algorithm. 
Place $v_{i+1}$ at point $\left(x\left(v_{i}\right)+2^{4 i+3}-2,0\right), h_{i+1}^{a}$ at point $\left(x\left(v_{i}\right)+2^{4 i+3}-3,2^{2 i+2}\right)$, and $a_{i+1}$ at point $\left(x\left(v_{i}\right)+2^{4 i+3}-4,2^{2 i+3}\right)$ (see Fig. 6, b). Such placements guarantee that part (ii) of the hypothesis is verified. The slope of segment $\overline{v_{i+1} a_{i+1}}$ is $-2^{2(i+1)} / 1$. Hence, the slope of its axis is $1 / 2^{2(i+1)}$. Such an axis passes through point $q_{i} \equiv\left(x\left(v_{i}\right)-\right.$ $\left.3,2^{2 i+1}\right)$. Since $0<1 / 2^{2(i+1)}<1 / 2^{2 i}$, it follows that path $\left(v_{1}, v_{2}, \ldots, v_{i}\right)$, together with nodes $a_{1}, a_{2}, \ldots, a_{i}$, lies below the axis of $\overline{v_{i+1} a_{i+1}}$. Finally, the axis of $\overline{v_{i} a_{i}}$ passes through point $p_{i+1} \equiv\left(x\left(v_{i}\right)+2^{4 i+3}-4,2^{2 i}+2^{2 i+3}-3 / 2^{2 i}\right)$. Thus, $y\left(p_{i+1}\right)>$ $y\left(a_{i+1}\right)$, since $2^{2 i}+2^{2 i+3}-3 / 2^{2 i}>2^{2 i+3}$ as long as $2^{4 i}>3$, which holds for each $i \geq 1$. This implies that part (i) of the hypothesis is verified.

When the algorithm has drawn $v_{n}$ and $a_{n}$ (and symmetrically $b_{n}$ ), $c_{n}$ and $d_{n}$ still have to be drawn. However, this can be easily done by assigning to segments $\overline{v_{n} c_{n}}$ and $\overline{v_{n} d_{n}}$ the same length as segment $\overline{v_{n} a_{n}}$ and by placing them so that the angle $\overline{b_{n} v_{n} a_{n}}$, which is strictly greater than $180^{\circ}$, is split into three angles strictly greater than $60^{\circ}$.

We remark that $c_{n}$ and $d_{n}$ are not placed at points with rational coordinates. However, they still obey to any resolution rule, namely their distance from any node or edge of the drawing is exponential with respect to the grid unit. Placing such nodes at grid points is possible after a scaling of the whole drawing and some non-trivial calculations. However, we preferred not to deal with such an issue since we just needed to prove that a greedy drawing of $T_{n}$ exists.

\section{Conclusions}

In this paper we have shown that constructing succinct greedy drawings in the plane, when the Euclidean distance is adopted as a metric, may be unfeasible even for simple classes of trees. In fact, we proved that there exist caterpillars requiring exponential area in any greedy drawing, under any finite resolution rule. The proof uses a mixed geometric-topological technique that allows us to analyze the combinatorial space of the possible embeddings and to identify invariants of the slopes of the edges in any greedy drawing of such caterpillars.

Many problems remain open in this area. By the results of Leighton and Moitra [9], every triconnected planar graph admits a greedy drawing.

Problem 1. Which are the area requirements of greedy drawings of triconnected planar graphs?

While every triconnected planar graph admits a greedy drawing, not all biconnected planar graphs and not all trees admit a greedy drawing. For example, in [9] it is shown that a complete binary tree with 31 nodes does not admit any greedy drawing. Hence, the following problem is worth studying:

Problem 2. Characterize the class of trees (resp. of biconnected planar graphs) that admit a greedy drawing.

In this paper we argued about the relationship among greedy drawings, planarity, and straight-line drawability. We have shown, in Lemma 4 that every straight-line greedy drawing of a tree is planar. It would be interesting to understand whether trees are the only class of planar graphs with such a property. 
Problem 3. Characterize the class of planar graphs such that every straight-line greedy drawing is planar.

\section{References}

1. Angelini, P., Frati, F., Grilli, L.: An algorithm to construct greedy drawings of triangulations. In: Tollis, I.G., Patrignani, M. (eds.) GD 2008. LNCS, vol. 5417, pp. 26-37. Springer, Heidelberg (2009)

2. Dhandapani, R.: Greedy drawings of triangulations. In: Huang, S.T. (ed.) SODA 2008, pp. 102-111 (2008)

3. Di Battista, G., Lenhart, W., Liotta, G.: Proximity drawability: a survey. In: Tamassia, R., Tollis, I.G. (eds.) GD 1994. LNCS, vol. 894, pp. 328-339. Springer, Heidelberg (1995)

4. Di Battista, G., Tamassia, R., Tollis, I.G.: Area requirement and symmetry display of planar upward drawings. Discrete \& Computational Geometry 7, 381-401 (1992)

5. Eppstein, D., Goodrich, M.T.: Succinct greedy graph drawing in the hyperbolic plane. In: Tollis, I.G., Patrignani, M. (eds.) GD 2008. LNCS, vol. 5417, pp. 14-25. Springer, Heidelberg (2009)

6. Kaufmann, M.: Polynomial area bounds for MST embeddings of trees. In: Hong, S.-H., Nishizeki, T., Quan, W. (eds.) GD 2007. LNCS, vol. 4875, pp. 88-100. Springer, Heidelberg (2008)

7. Kleinberg, R.: Geographic routing using hyperbolic space. In: INFOCOM 2007, pp. 1902 1909 (2007)

8. Knaster, B., Kuratowski, C., Mazurkiewicz, C.: Ein beweis des fixpunktsatzes fur n dimensionale simplexe. Fundamenta Mathematicae 14, 132-137 (1929)

9. Leighton, T., Moitra, A.: Some results on greedy embeddings in metric spaces. In: FOCS 2008, pp. 337-346 (2008)

10. Monma, C.L., Suri, S.: Transitions in geometric minimum spanning trees. Discrete \& Computational Geometry 8, 265-293 (1992)

11. Papadimitriou, C.H., Ratajczak, D.: On a conjecture related to geometric routing. Theoretical Computer Science 344(1), 3-14 (2005)

12. Penna, P., Vocca, P.: Proximity drawings in polynomial area and volume. Computational Geometry 29(2), 91-116 (2004)

13. Rao, A., Papadimitriou, C.H., Shenker, S., Stoica, I.: Geographic routing without location information. In: Johnson, D.B., Joseph, A.D., Vaidya, N.H. (eds.) MOBICOM 2003, pp. 96 108 (2003)

14. Schnyder, W.: Embedding planar graphs on the grid. In: SODA 1990, pp. 138-148 (1990) 